\title{
ON THE MULTIPLE VALUES OF ALGEBROID FUNCTIONS*
}

\author{
ZONGSHENG GAO
}

\begin{abstract}
For any $v$-valued algebroid function of finite order $\rho>0$ in $|z|<\infty$, we prove the existence of the sequence of filling disks and Borel direction dealing with its multiple values.
\end{abstract}

\section{Introduction}

Valiron [1] conjectured that there exists at least a Borel direction for any $v$ valued algebroid function of order $\rho(0<\rho<\infty)$. Rauch [2] proved that there exists a direction such that the corresponding Borel exceptional values form a set of linear measure zeros. Toda [3] proved that there exists a direction such that the set of corresponding Borel exceptional values is countable. Later Lü and $\mathrm{Gu}$ [4] proved that there exists a direction such that the number of Borel exceptional values is equal to $2 v$ at most. However, it was not discussed whether there exists a Borel direction dealing with its multiple values. In the present paper we investigate this problem.

Let $w=w(z)$ be a $v$-valued algebroid function in $|z|<\infty$ defined by irreducible equation

$$
A_{v}(z) w^{v}+A_{v-1}(z) w^{v-1}+\cdots+A_{0}(z)=0,
$$

where $A_{v}(z), \ldots, A_{0}(z)$ are entire functions without any common zero. The single valued domain of definition of $w(z)$ is a $v$-sheeted covering of $z$-plane, a Riemann surface, denoted by $\tilde{R}_{z}$. A point in $\tilde{R}_{z}$ whose projection in the $z$-plane is $z$, is denoted by $\tilde{z}$. The part of $\tilde{R}_{z}$, which covers a disk $|z|<r$, is denoted by $|\tilde{z}|<r$. Let $n(r, a)$ be the number of the zeros, counted according to their multiplicities, of $w(z)-a$ in $|\tilde{z}| \leq r, \bar{n}^{l)}(r, a)$ be the number of distinct zeros with multiplicity $\leq l$ of $w(z)-a$ in $|\tilde{z}| \leq r$. Let

1991 Mathematics Subject Classification: 30 D35.

Key words: Algebroid function, Multiple Value, Borel direction.

* Project supported by the National Natural Science Foundation of China.

Received May 30, 1997; revised February 8, 1999. 


$$
\begin{aligned}
& S(r, w)=\frac{1}{\pi} \iint_{|z| \leq r}\left(\frac{\left|w^{\prime}(z)\right|}{1+|w(z)|^{2}}\right)^{2} d \omega, \\
& T(r, w)=\frac{1}{v} \int_{0}^{r} \frac{S(r, w)}{r} d r .
\end{aligned}
$$

$S(r, w)$ is called the mean covering number of $|\tilde{z}| \leq r$ into $w$-sphere under the mapping $w=w(z) . T(r, w)$ is called the characteristic function of $w(z)$. Let

$$
\begin{aligned}
& N(r, a)=\frac{1}{v} \int_{0}^{r} \frac{n(r, a)-n(0, a)}{r} d r+\frac{n(0, a)}{v} \log r, \\
& m(r, w)=\frac{1}{2 \pi v} \int_{|\tilde{z}|=r} \log ^{+}\left|w\left(r e^{i \theta}\right)\right| d \theta, \quad z=r e^{i \theta},
\end{aligned}
$$

where $|\tilde{z}|=r$ is the boundary of $|\tilde{z}| \leq r$. We have

$$
T(r, w)=m(r, w)+N(r, \infty)+O(1) .
$$

The order of algebroid function $w(z)$ is defined by

$$
\rho=\varlimsup_{r \rightarrow \infty} \frac{\log ^{+} T(r, w)}{\log r} .
$$

In this paper, we suppose that $0<\rho<\infty$. Let $n\left(r, \tilde{R}_{z}\right)$ be the number of the branch points of $\tilde{R}_{z}$ in $|\tilde{z}| \leq r$, counted with the order of branch. Write

$$
N\left(r, \tilde{R}_{z}\right)=\frac{1}{v} \int_{0}^{r} \frac{n\left(r, \tilde{R}_{z}\right)-n\left(0, \tilde{R}_{z}\right)}{r} d r+\frac{n\left(0, \tilde{R}_{z}\right)}{v} \log r .
$$

By [5]

$$
N\left(r, \tilde{R}_{z}\right) \leq 2(v-1) T(r, w)+O(1) .
$$

We define angular domain:

$$
\Delta\left(\theta_{0}, \delta\right)=\left\{z|| \arg z-\theta_{0} \mid<\delta\right\}, \quad 0 \leq \theta_{0}<2 \pi, \quad 0<\delta<\frac{\pi}{2} .
$$

The part of $\tilde{R}_{z}$ which lie over $\Delta\left(\theta_{0}, \delta\right)$ is denoted by $\tilde{\Delta}\left(\theta_{0}, \delta\right)$. Let $n\left(r, \Delta\left(\theta_{0}, \delta\right), a\right)$ be the number of the zeros of $w(z)-a$ in $\tilde{\Delta}\left(\theta_{0}, \delta\right) \cap\{|\tilde{z}| \leq r\}$ and $n\left(r, \Delta\left(\theta_{0}, \delta\right), \tilde{R}_{z}\right)$ be the number of the branch points in the same region. Similarly as above, we can define $\bar{n}^{l)}\left(r, \Delta\left(\theta_{0}, \delta\right), a\right)$.

DEFINITION. Let $w=w(z)$ be a $v$-valued algebroid function of order $\rho(0<\rho<\infty)$ defined by $(1)$ in $|z|<\infty$, and $l \geq 3$ be a position integer. For arbitrary $\delta>0(0<\delta<\pi / 2)$, if

$$
\varlimsup_{r \rightarrow \infty} \frac{\log ^{+} \bar{n}^{l)}\left(r, \Delta\left(\theta_{0}, \delta\right), a\right)}{\log r}=\rho
$$


holds for any complex value a except at most finite possible exceptions, then the half line $B: \arg z=\theta_{0}\left(0 \leq \theta_{0}<2 \pi\right)$ is called a Borel direction about multiple values of $w(z)$.

In this paper, the Riemann sphere of diamte 1 is denoted by $V, C$ is a positive constant and it may be of different meaning when it appears in different position.

\section{Some lemmas}

Let $F_{1}$ be a connected domain on $V$, the boundary of $F_{1}$ is denoted by $\partial F_{1}$, which consists of a finite number of mutually disjoint circular curves $\left\{\Lambda_{j}\right\}$, and the spherical distance between any two circular curves $\wedge_{l}$ and $\wedge_{J}$ is $d\left(\wedge_{l}, \wedge_{J}\right) \geq$ $\delta \in(0,1 / 2)(i \neq j)$.

Let $F$ be a finite covering surface of $F_{1}, F$ is bounded by a finite number of analytic closed Jordan curves, its boundary is denoted by $\partial F$. We call the part of $\partial F$, which lies the interior of $F_{1}$, the relative boundary of $F$, and denote its length by $L$.

Let $D$ be a domain on $F_{1}$, its boundary consists of a finite number of points or analytic closed Jordan curves, and $F(D)$ be the part of $F$, which lies above $D$. We denote the area of $F, F_{1}, F(D)$ and $D$ by $|F|,\left|F_{1}\right|,|F(D)|$ and $|D|$ respectively. We call

$$
S=\frac{|F|}{\left|F_{1}\right|}, \quad S(D)=\frac{|F(D)|}{|D|}
$$

the mean covering number of $F$ relative to $F_{1}, D$ respectively.

Under the above hypotheses, Sun Daochun [6] estimated the constants of Ahlfors' covering theorem and Ahlfors' fundamental theorem [7] on unit sphere, and obtained the following results:

LEMMA 1.

$$
|S-S(D)|<\max \left\{\frac{2}{\delta}, \frac{\pi^{2}}{|D|}\right\} L .
$$

LEMMA 2 .

$$
\rho^{+}(F) \geq \rho\left(F_{1}\right) S-2^{5} \pi^{2} \delta^{-3} L\left(F_{1}\right),
$$

where $\rho(F), \rho\left(F_{1}\right)$ is Eulers' characteristic of $F, F_{1}$ respectively, $\rho^{+}=\max \{\rho, 0\}$, $L\left(F_{1}\right)$ is the length of relative boundary of $F$ with respect to $F_{1}$.

Let $D_{j}(j=1,2, \ldots, q)$ be $q(q \geq 3)$ disjoint circles on $V, d\left(D_{i}, D_{j}\right) \geq \delta \in$ $(0,1 / 2)(i \neq j)$.

We take off $\left\{D_{j}\right\}$ from $V$ and let $F_{0}$ be the remaining surface, then $\rho\left(F_{0}\right)=q-2$. 
Now $F\left(D_{j}\right)$ consists of a finite number of connected surfaces

$$
F\left(D_{j}\right)=\bigcup_{k} F_{J, k}^{l}+\bigcup_{k} F_{J, k}^{z},
$$

where $F_{J, k}$ has no relative boundary, with respect to $D_{j}$, which is called an island and $F_{J, k}^{z}$ has such one, which is called a peninsula.

In the following, we shall give two Lemmas for later use, their proof methods belong to Tsuji [7].

LEMMA 3. Let $F$ be $m$ connected covering surfaces on the unit sphere $V$, $\rho(F)=\tilde{n}-m(\tilde{n}$ is a nonegative integer $),\left\{D_{v}\right\}$ be $q(q \geq 3)$ disjoint disks on $V$, where the spherical distance $d\left(D_{i}, D_{j}\right) \geq \delta \in(0,1 / 2)(i \neq j)$.

If $n_{j}$ is the number of simply connected islands in $F\left(D_{j}\right)$, then

$$
\tilde{n}+\sum_{j=1}^{q} n_{j} \geq(q-2) S-\frac{C}{\delta^{3}} L,
$$

where $C>0$ is a constant and $L$ is the length of the boundary of $F$.

Proof. We take off from $F$ all peninsulas $\left\{F_{j, k}^{z}\right\}$ above $\left\{D_{j}\right\}$, and let $F^{\prime}$ be the remaining surface:

$$
F^{\prime}=F-\bigcup_{j=1}^{q} \bigcup_{k} F_{J, k}^{z}
$$

Since the peninsulas involve only the part of the boundary of $F$, then $\rho\left(F^{\prime}\right) \leq \rho(F)$. Suppose that $F^{\prime}$ consists of $N\left(F^{\prime}\right)$ of connected surfaces, then $N\left(F^{\prime}\right) \leq m$. Hence

$$
\rho\left(F^{\prime}\right) \leq \rho(F)=\tilde{n}-m \leq \tilde{n}-N\left(F^{\prime}\right) .
$$

Next we take off from $F^{\prime}$ all islands $\left\{F_{j, k}^{l}\right\}$ above $\left\{D_{j}\right\}$ and let $F^{\prime \prime}$ be the remaining surface:

$$
F^{\prime}=\bigcup_{j=1}^{q} \bigcup_{k} F_{J, k}^{l}+F^{\prime \prime} .
$$

$F^{\prime \prime}$ consists of a finite number of connected surfaces:

$$
F^{\prime \prime}=\bigcup_{\mu} F_{\mu}^{\prime \prime}
$$

So that

$$
F^{\prime}=\bigcup_{J=1}^{q} \bigcup_{k} F_{J, k}^{l}+\bigcup_{\mu} F_{\mu}^{\prime \prime} .
$$

Since $F^{\prime}$ is decomposed into $\left\{F_{J, k}^{l}\right\}$ and $\left\{F_{\mu}^{\prime \prime}\right\}$ by ring cuts, so its characteristic not change. 
Hence

$$
\rho\left(F^{\prime}\right)=\sum_{J=1}^{q} \sum_{k} \rho\left(F_{J, k}^{l}\right)+\sum_{\mu} \rho\left(F_{\mu}^{\prime \prime}\right)
$$

By (4)

$$
\tilde{n}-N\left(F^{\prime}\right) \geq \rho\left(F^{\prime}\right)=\sum_{j=1}^{q} \sum_{k} \rho\left(F_{J, k}^{l}\right)+\sum_{\mu} \rho^{+}\left(F_{\mu}^{\prime \prime}\right)-N\left(F^{\prime \prime}\right),
$$

where $N\left(F^{\prime \prime}\right)$ is the number of simply connected $F_{\mu}^{\prime \prime}$.

Since

$$
\sum_{j=1}^{q} \sum_{k} \rho\left(F_{J, k}^{l}\right)=\sum_{j=1}^{q} \sum_{k} \rho^{+}\left(F_{J, k}^{l}\right)-\sum_{j=1}^{q} n_{j}
$$

so by (5), (6) we have

$$
\begin{aligned}
\sum_{J=1}^{q} n_{j} & =\sum_{j=1}^{q} \sum_{k} \rho^{+}\left(F_{J, k}^{l}\right)-\sum_{j=1}^{q} \sum_{k} \rho\left(F_{J, k}^{l}\right) \\
& \geq \sum_{J=1}^{q} \sum_{k} \rho^{+}\left(F_{J, k}^{l}\right)+\sum_{\mu} \rho^{+}\left(F_{\mu}^{\prime \prime}\right)-N\left(F^{\prime \prime}\right)+N\left(F^{\prime}\right)-\tilde{n} .
\end{aligned}
$$

We see easily that $N\left(F^{\prime}\right)-N\left(F^{\prime \prime}\right) \geq 0$, hence

$$
\tilde{n}+\sum_{j=1}^{q} n_{j} \geq \sum_{\mu} \rho^{+}\left(F_{\mu}^{\prime \prime}\right) .
$$

Put $F_{0}=V-\bigcup_{v=1}^{q} D_{v}$, then $F_{\mu}^{\prime \prime}$ is a covering surface of $F_{0}$, by Lemma 2,

$$
\rho^{+}\left(F_{\mu}^{\prime \prime}\right) \geq(q-2) S_{\mu}^{\prime \prime}-\frac{2^{5} \pi^{2}}{\delta^{3}} L_{\mu}^{\prime \prime}
$$

where $S_{\mu}^{\prime \prime}=\left|F_{\mu}^{\prime \prime}\right| /\left|F_{0}\right|$ and $L_{\mu}^{\prime \prime}$ is the length of the relative boundary of $F_{\mu}^{\prime \prime}$ with respect to $F_{0}$.

By (8), (9)

$$
\begin{aligned}
\tilde{n}+\sum_{j=1}^{q} n_{j} & \geq \sum_{\mu}(q-2) S_{\mu}^{\prime \prime}-\sum_{\mu} \frac{2^{5} \pi^{2}}{\delta^{3}} L_{\mu}^{\prime \prime} \\
& =(q-2) S^{\prime \prime}-\frac{2^{5} \pi^{2}}{\delta^{3}} L^{\prime \prime},
\end{aligned}
$$

where $S^{\prime \prime}=\sum_{\mu} S_{\mu}^{\prime \prime}=\left|F^{\prime \prime}\right| /\left|F_{0}\right|, L^{\prime \prime}=\sum_{\mu} L_{\mu}^{\prime \prime}$.

By Lemma $1,\left|S-S^{\prime \prime}\right|<\max \left\{2 / \delta, \pi^{2} /\left|F_{0}\right|\right\} L^{\prime \prime}$.

Since $\left|F_{0}\right|<|V|=\pi$, then $\pi^{2} /\left|F_{0}\right| \geq 1$, so that $\left|S-S^{\prime \prime}\right|<(2 / \delta)\left(\pi^{2} /\left|F_{0}\right|\right) L^{\prime \prime}$. 
Since $(\delta / 2)^{2} q \leq\left|F_{0}\right|, L^{\prime \prime} \leq L$, we have

$$
S^{\prime \prime}>S-\frac{2^{3} \pi^{2}}{\delta^{3} q} L
$$

Hence by (10), (11)

$$
\tilde{n}+\sum_{j=1}^{q} n_{j} \geq(q-2) S-\frac{C}{\delta^{3}} L
$$

where $C=40 \pi^{2}$.

Lemma 4. Under the same condition as in Lemma 3 , let $D_{j}(j=1,2, \ldots, q)$ be $q(q \geq 3)$ disjoint disks with radius $\delta / 3$, and $n_{j}^{l)}$ be the number of simply connected islands in $F\left(D_{j}\right)$, which consist of not more than $l$ sheets, then

$$
(l+1) \tilde{n}+l \sum_{j=1}^{q} n_{j}^{l)} \geq(l+1)(q-2) S-\frac{C}{\delta^{5}} L,
$$

where $l \geq 3$ is a positive integer.

Proof. Let $n_{j}$ be the number of simply connected islands in $F\left(D_{j}\right)$, and $n_{j}^{(l}$ be that of such ones, which consist of more than $l$ sheets, then

$$
n_{j}=n_{j}^{l)}+n_{j}^{(l}, \quad S\left(D_{j}\right) \geq n_{j}^{l)}+(l+1) n_{j}^{(l},
$$

so that

$$
S\left(D_{j}\right) \geq(l+1)\left(n_{j}^{l)}+n_{j}^{(l)}-\ln _{j}^{l)}=(l+1) n_{j}-\ln _{j}^{l}\right) .
$$

Since $\left|D_{j}\right| \geq \delta^{2} / 9$, from Lemma 1 , we have

$$
S+\frac{18 \pi^{2}}{\delta^{3}} L>S\left(D_{j}\right)
$$

hence by $(12), S+\left(18 \pi^{2} / \delta^{3}\right) L>(l+1) n_{j}-\ln _{j}^{l)}$, so that

$$
q S+l \sum_{j=1}^{q} n_{j}^{l)}+\frac{18 \pi^{2}}{\delta^{3}} q L>(l+1) \sum_{j=1}^{q} n_{j} .
$$

Note that $q(\delta / 2)^{2} \leq \pi$, by Lemma 3 and (13) we have

$$
(l+1) \tilde{n}+l \sum_{j=1}^{q} n_{j}^{l)} \geq(l+1)(q-2) S-\frac{C}{\delta^{5}} L .
$$

\section{A fundamental inequality of algebroid functions}

THEOREM 1. Let $w=w(z)$ be a v-valued algebroid function in $|z|<R$ $(0<R<\infty), F$ be the Riemann surface, generated by $w=w(z)$ on the $w$-sphere $V$, 
and $D_{1}, D_{2}, \ldots, D_{q}$ be $q(q \geq 3)$ disjoint disks with radius $\delta / 3$ on $V, d\left(D_{i}, D_{j}\right) \geq \delta \in$ $(0,1 / 2)(i \neq j)$. Suppose that $n_{j}^{l)}$ is the number of simply connected islands in $F\left(D_{j}\right)$, which consist of not more than $l$ sheets, then for any $r \in(0, R)$

$$
(q-2) S(r) \leq \sum_{j=1}^{q} n_{j}^{l)}+n\left(R, \tilde{R}_{z}\right)+\frac{C}{\delta^{25}} \frac{R}{R-r},
$$

where $l \geq 3$ is a positive integer, $n\left(R, \tilde{R}_{z}\right)$ is as in section 1 .

Proof. We take off $D_{1}, D_{2}, \ldots, D_{q}$ from the $w$-sphere $V$ and let $F_{0}$ be the remaining surface, then $\rho\left(F_{0}\right)=q-2$.

Let $\tilde{D}_{r}=\{|\tilde{z}|<r\}(r \in(0, R))$ be the part of $\tilde{R}_{z}$ in $|z|<r$, then by M. Hurwitz formula [8]

$$
\rho\left(\tilde{D}_{r}\right)=n\left(r, \tilde{R}_{z}\right)-v .
$$

From Lemma 4, we easily obtain

$$
(l+1) n\left(r, \tilde{R}_{z}\right)-(l+1) v+l \sum_{J=1}^{q} n_{J}^{l)} \geq(l+1)(q-2) S(r)-\frac{C}{\delta^{5}} L(r),
$$

where

$$
\begin{gathered}
S(r)=\frac{1}{\pi} \iint_{|\tilde{z}| \leq r}\left(\frac{\left|w^{\prime}(z)\right|}{1+|w(z)|^{2}}\right)^{2} d \omega, \\
L(r)=\int_{|\tilde{z}|=r} \frac{\left|w^{\prime}\left(r e^{\imath \varphi}\right)\right|}{1+\left|w\left(r e^{\imath \varphi}\right)\right|^{2}} r d \varphi .
\end{gathered}
$$

By Schwarz's inequality,

$$
L^{2}(r) \leq 2 \pi^{2} v r \frac{d S(r)}{d r}
$$

Put $\sum_{J=1}^{q} n_{j}^{l)}+n\left(r, \tilde{R}_{z}\right) \stackrel{\text { def }}{=} N$, from (14), we have

$$
N \geq(q-2) S(r)-\frac{C}{\delta^{5}} L(r) .
$$

If $(q-2) S\left(r^{\prime}\right)-N>0$ for all $r^{\prime} \in(r, R)$, then from (15), we have

$$
\left((q-2) S\left(r^{\prime}\right)-N\right)^{2} \leq \frac{C}{\delta^{25}} L^{2}\left(r^{\prime}\right) \leq \frac{2 \pi^{2} v R C}{\delta^{25}} \frac{d S\left(r^{\prime}\right)}{d r^{\prime}}
$$

so that

$$
R-r=\int_{r}^{R} d r^{\prime} \leq \frac{C}{\delta^{25}} R \int_{r}^{R} \frac{d S\left(r^{\prime}\right)}{\left[(q-2) S\left(r^{\prime}\right)-N\right]^{2}} \leq \frac{C}{\delta^{25}} \frac{R}{(q-2) S(r)-N}
$$


From this, we have

$$
(q-2) S(r) \leq N+\frac{C}{\delta^{25}} \frac{R}{R-r}=\sum_{j=1}^{q} n_{j}^{l)}+n\left(r, \tilde{R}_{z}\right)+\frac{C}{\delta^{25}} \frac{R}{R-r} .
$$

If $(q-2) S\left(r^{\prime}\right)-N \leq 0$ for some $r^{\prime} \in(r, R)$, then $(q-2) S\left(r^{\prime}\right) \leq N$, so that (16) holds in general.

This completes the proof of Theorem 1 .

Applying Theorem 1, we have the following

COROllary 1. Let $w=w(z)$ be a v-valued algebroid function in $|z|<R$, and $a_{1}, a_{2}, \ldots, a_{q}(q \geq 3)$ be $q$ disjoint points on $w$-sphere $V$, where the spherical distances between any two of them satisfy $d\left(a_{i}, a_{j}\right) \geq \delta \in(0,1 / 2)(i \neq j)$, then for any $r \in(0, R)$, we have

$$
(q-2) S(r) \leq \sum_{J=1}^{q} \bar{n}^{l)}\left(R, a_{j}\right)+n\left(R, \tilde{R}_{z}\right)+\frac{C}{\delta^{25}} \frac{R}{R-r} .
$$

where $l \geq 3$ is a positive integer, $\bar{n}^{l)}\left(R, a_{j}\right)$ is as in section 1 .

\section{The sequence of filling disks for algebroid functions}

LEMMA 5. Let $w=w(z)$ be a v-valued algebroid function of order $\rho$ $(0<\rho<\infty)$ defined by (1) in $|z|<\infty$, and $l \geq 3$ be a positive integer. For arbitrarily constants $\varepsilon \in(0, \rho)$ and $R>1$, there exists $a_{0} \in(1,2)$ such that for any $a \in\left(1, a_{0}\right)$ the following assertion is true:

Set $r_{n}=a^{n}$ and $m=\left[2 \pi r_{n-1} /\left(r_{n}-r_{n-1}\right)\right]=[2 \pi /(a-1)]$, where $[x]$ is the integral part of $x$. For integers $p, q$ with $p>0$ and $0 \leq q<m$, let $\theta_{q}=$ $2 \pi(q+1) / m$, let $\Omega_{p, q}$ be the domain $\left\{a^{p-1} \leq|z|<a^{p+2}\right\} \cap\left\{\left|\arg z-\theta_{q}\right| \leq 2 \pi / m\right\}$ and let $\bar{n}^{l)}\left(\Omega_{p, q}, w=\alpha\right)$ be the number of distinct zeros with multiplicity $\leq l$ of $w(z)-\alpha$ in $\tilde{\Omega}_{p, q}$. Then there exist at least a pair of integers $p_{0}, q_{0}$, with $a^{p_{0}}>R$, and $(l v-1)$ domains enclosed by spherical circles of radius $\delta=a^{-p_{0} \rho / 26}$ on the Riemann sphere such that $\bar{n}^{l)}\left(\Omega_{p_{0}, q_{0}}, w=\alpha\right) \geq a^{p_{0}(\rho-\varepsilon)}$ for any complex value $\alpha$ not in the $(l v-1)$ domains.

Proof. Suppose that the conclusion is false. Then, for some $\varepsilon \in(0, \rho)$ and $R>1$, and any given sequence $\left\{a_{i}\right\}$ with $a_{i}>1$ and $a_{i} \rightarrow 1(i \rightarrow \infty)$, there exists at least a point $a \in\left(1, a_{i}\right)$ for each $i$, such that to any integers $p>$ $P \stackrel{\text { def }}{=}[\log R / \log a]$ and $q \in\{0,1,2, \ldots, m-1\}$, there exist accordingly $l v$ complex numbers $\left\{\alpha_{j}=\alpha_{j}(p, q)\right\}_{j=1}^{l v}$ such that

$$
\bar{n}^{l)}\left(\Omega_{p, q}, w=\alpha_{j}\right)<a^{p(\rho-\varepsilon)},
$$

where the spherical distance $d\left(\alpha_{j}, \alpha_{k}\right) \geq \delta=a^{-p \rho / 26}(j \neq k)$.

Taking $r>R$ arbitrarily, set $T=[\log r / \log a]\left(a^{T} \leq r<a^{T+1}\right)$. For any positive integers $M$ and $N$, put 


$$
\begin{aligned}
b & =a^{\frac{1}{M}}, r_{p, t}=b^{M p+t}, \quad t=0,1,2, \ldots, M-1, \\
L_{p, t} & =\left\{r_{p, t} \leq|z|<r_{p, t+1}\right\}, \quad \theta_{q, J}=\frac{2 \pi q}{m}+\frac{2 \pi j}{N m}, \\
\Delta_{q, J} & =\left\{z ;|z|<a^{T}, \theta_{q, J} \leq \arg z<\theta_{q, J+1}\right\} .
\end{aligned}
$$

Since

$$
\begin{aligned}
\left\{a^{-1} \leq|z|<a^{T}\right\} & =\bigcup_{t=0}^{M-1} \bigcup_{p=-1}^{T-1} L_{p, t} \\
\left\{|z|<a^{T}\right\} & =\bigcup_{J=0}^{N-1} \bigcup_{q=0}^{m-1} \Delta_{q, J}
\end{aligned}
$$

then there certainly exist a pair of $t_{0}, j_{0}$, which depend on $T$, without loss of generality, we may assume that $t_{0}=0, j_{0}=0$, such that

$$
\begin{aligned}
& \sum_{p=-1}^{T-1} n\left(L_{p, 0}, \tilde{R}_{z}\right) \leq \frac{1}{M} n\left(a^{T}, \tilde{R}_{z}\right), \\
& \sum_{q=0}^{m-1} n\left(\Delta_{q, 0}, \tilde{R}_{z}\right) \leq \frac{1}{N} n\left(a^{T}, \tilde{R}_{z}\right),
\end{aligned}
$$

where $n\left(L_{p, 0}, \tilde{R}_{z}\right)$ and $n\left(\Delta_{q, 0}, \tilde{R}_{z}\right)$ are the number of branch points of $\tilde{R}_{z}$ in $\tilde{L}_{p, 0}$ and $\tilde{\Delta}_{q, 0}$ respectively.

Put

$$
\begin{gathered}
\Omega_{p, q}^{0}=\left\{\frac{b^{M p}+b^{M p+1}}{2} \leq|z|<\frac{b^{M p+M}+b^{M p+M+1}}{2}\right\} \\
\cap\left\{\frac{\theta_{q, 0}+\theta_{q, 1}}{2} \leq \arg z<\frac{\theta_{q+1,0}+\theta_{q+1,1}}{2}\right\}, \\
\bar{\Omega}_{p, q}=\left\{b^{M p} \leq|z|<b^{M p+M+1}\right\} \cap\left\{\theta_{q, 0} \leq \arg z<\theta_{q+1,1}\right\} .
\end{gathered}
$$

Then

$$
\Omega_{p, q}^{0} \subset \bar{\Omega}_{p, q} \subset \Omega_{p, q} .
$$
we have

Since $\left\{\bar{\Omega}_{p, q}\right\}$ overlap $\bigcup_{p=-1}^{T-1} L_{p, 0}$ and $\bigcup_{q=0}^{m-1} \Delta_{q, 0}$ twice at most, from (18), (19)

$$
\sum_{p=1}^{T-2} \sum_{q=0}^{m-1} n\left(\bar{\Omega}_{p, q}, \tilde{R}_{z}\right) \leq\left(1+\frac{1}{M}+\frac{1}{N}\right) n\left(a^{T}, \tilde{R}_{z}\right),
$$

where $n\left(\bar{\Omega}_{p, q}, \tilde{R}_{z}\right)$ is the number of branch points of $\tilde{R}_{z}$ in $\tilde{\bar{\Omega}}_{p, q}$.

Obviously, $\bar{\Omega}_{p, q}$ can be mapped conformally to unit disk $|\zeta|<1$ such that the center of $\Omega_{p, q}^{0}$ corresponds to $\zeta=0$ and the image of $\Omega_{p, q}^{0}$ is contained in the 
disk $|\zeta|<\kappa<1$, where $\kappa>0$ is a constant, independent of $p$ and $q$. Hence by Corollary 1 and (17), (20), we have

$$
\begin{aligned}
(l v-2) S\left(a^{T-1}, w\right) \leq & (l v-2) \sum_{p=P+1}^{T-2} \sum_{q=0}^{m-1}\left(S\left(\Omega_{p, q}^{0}, w\right)+(l v-2) S\left(a^{P+2}, w\right)\right. \\
\leq & \sum_{p=P+1}^{T-2} \sum_{q=0}^{m-1}\left(\sum_{J=1}^{l v} \bar{n}^{l)}\left(\bar{\Omega}_{p, q}, w=\alpha_{J}\right)+n\left(\bar{\Omega}_{p, q}, \tilde{R}_{z}\right)+\frac{C}{\delta^{25}} \frac{1}{1-\kappa}\right) \\
& +(l v-2) S\left(a^{P+2}, w\right) \\
\leq & l v T m a^{(T-1)(\rho-\varepsilon)}+\left(1+\frac{1}{M}+\frac{1}{N}\right) n\left(a^{T}, \tilde{R}_{z}\right) \\
& +\operatorname{Tm} \frac{C}{1-\kappa}\left(a^{T \rho / 26}\right)^{25}+(l v-2) S\left(a^{P+2}, w\right),
\end{aligned}
$$

where $S\left(\Omega_{p_{0}, q_{0}}^{0}, w\right)=1 / \pi \iint_{\tilde{\Omega}_{p_{0}, q_{0}}^{0}}\left(\left|w^{\prime}(z)\right| /\left(1+|w(z)|^{2}\right)^{2} d \omega, \bar{n}^{l)}\left(\bar{\Omega}_{p, q}, w=\alpha_{j}\right)\right.$ is the number of distinct zeros with multiplicity $\leq l$ of $w(z)-\alpha_{j}$ in $\tilde{\bar{\Omega}}_{p, q}$. Taking $T(=[\log r / \log a])$ sufficiently large, then $r$ sufficiently large too, and $r \in$ $\left[a^{T}, a^{T+1}\right)$, thus we have

$$
(l v-2) S\left(r a^{-2}, w\right) \leq r^{\rho-(\varepsilon / 2)}+\left(1+\frac{1}{M}+\frac{1}{N}\right) n\left(r, \tilde{R}_{z}\right)+C r^{(25 / 26) \rho}+C .
$$

Dividing this inequality by $r$ and integrating it, then

$$
(l v-2) T\left(r a^{-2}, w\right) \leq r^{\rho-(\varepsilon / 2)}+\left(1+\frac{1}{M}+\frac{1}{N}\right) N\left(r, \tilde{R}_{z}\right)+C r^{(25 / 26) \rho}+C \log r .
$$

From (2), we have

$$
\begin{gathered}
(l v-2) T\left(r a^{-2}, w\right) \leq \\
r^{\rho-(\varepsilon / 2)}+\left(1+\frac{1}{M}+\frac{1}{N}\right)(2 v-2) T(r, w) \\
+O(1)+C r^{(25 / 26) \rho}+C \log r .
\end{gathered}
$$

Suppose that $\rho(r)$ is a precise order of $T(r, w)$. Put $U(r)=r^{\rho(r)}$, then $\lim _{r \rightarrow \infty} \rho(r)=\rho$ and

$$
\lim _{r \rightarrow \infty} \frac{U\left(r a^{-2}\right)}{U(r)}=a^{-2 \rho}, \quad \varlimsup_{r \rightarrow \infty} \frac{T(r, w)}{U(r)}=1 .
$$

Dividing (21) by $U(r)$ and letting $r \rightarrow \infty$, we obtain

$$
(l v-2) a^{-2 \rho} \leq\left(1+\frac{1}{M}+\frac{1}{N}\right)(2 v-2) .
$$


Since $a \in\left(1, a_{i}\right)$, thus

$$
l v-2 \leq\left(1+\frac{1}{M}+\frac{1}{N}\right) a_{i}^{2 \rho}(2 v-2)
$$

Letting $i, M$ and $N \rightarrow \infty$ respectively, we have

$$
l v \leq 2 v \text {. }
$$

This is contrary to the condition $l \geq 3$, and Lemma 5 is proved.

THEOREM 2. Let $w=w(z)$ be a v-valued algebroid function of order $\rho$ $(0<\rho<\infty)$ defined by (1) in $|z|<\infty$, and $l \geq 3$ be a positive integer. Then there exists a sequence of filling disks for $w(z)$

$$
\begin{gathered}
\Gamma_{n}:\left\{\left|z-z_{n}\right|<r_{n} \sigma_{n}\right\}, \quad n=1,2, \ldots \\
z_{n}=r_{n} e^{i \theta_{n}}, \quad \lim _{n \rightarrow \infty} r_{n}=\infty, \quad \lim _{n \rightarrow \infty} \sigma_{n}=0\left(\sigma_{n}>0\right),
\end{gathered}
$$

such that for any complex value $\alpha$,

$$
\bar{n}^{l)}\left(\Gamma_{n}, w=\alpha\right) \geq r_{n}^{\rho-\varepsilon_{n}},
$$

with some possible exceptions for $\alpha$ enclosed in $l v-1$ spherical circles with radius $r_{n}^{-\rho / 26}$ and $\varepsilon_{n} \rightarrow 0(n \rightarrow \infty)$.

Proof. Let $\varepsilon_{n}=\rho / 2^{n}, R_{n}=2^{n}$. By Lemma 5, we have

$$
a_{n} \in\left(1,1+\frac{1}{n}\right), \quad m_{n}=\left[\frac{2 \pi}{a_{n}-1}\right], \quad p_{n}, \quad q_{n}, \quad \theta_{q_{n}}=\frac{2 \pi\left(q_{n}+1\right)}{m_{n}}
$$

and

$$
\Omega_{p_{n}, q_{n}}=\left\{a_{n}^{p_{n}-1} \leq|z| \leq a_{n}^{p_{n}+2}\right\} \cap\left\{\left|\arg z-\theta_{q_{n}}\right| \leq \frac{2 \pi}{m_{n}}\right\}(n=1,2, \ldots) .
$$

Let $\theta_{n}=\theta_{q_{n}}, z_{n}=a_{n}^{p_{n}} e^{i \theta_{n}}$, then $r_{n}=\left|z_{n}\right|=a_{n}^{p_{n}}>R_{n}=2^{n} \rightarrow \infty(n \rightarrow \infty)$.

Take $\sigma_{n}=4\left(a_{n}-1\right) \in(0,4 / n)$, then $\sigma_{n} \rightarrow 0(n \rightarrow \infty)$.

Let $\Gamma_{n}=\left\{\left|z-z_{n}\right|<\sigma_{n} r_{n}\right\}$, then $\Gamma_{n} \supset \Omega_{p_{n}, q_{n}}$.

Hence for any complex value $\alpha$, we have

$$
\bar{n}^{l)}\left(\Gamma_{n}, w=\alpha\right) \geq \bar{n}^{l)}\left(\Omega_{p_{n}, q_{n}}, w=\alpha\right) \geq r_{n}^{\rho-\varepsilon_{n}}
$$

with some possible exceptions for $\alpha$ enclosed in $l v-1$ spherical circles with radius $\delta=r_{n}^{-\rho / 26}$ and $\varepsilon_{n} \rightarrow 0(n \rightarrow \infty)$.

This proves Theorem 2 .

\section{The Borel direction of algebroid functions}

THEOREM 3. Suppose that $w=w(z)$ is a v-valued algebroid function of order $\rho(0<\rho<\infty)$ defined by (1) in $|z|<\infty, l \geq 3$ is a positive integer, 
then there exists a direction $B: \arg z=\theta_{0}\left(0 \leq \theta_{0}<2 \pi\right)$ such that for any given $\delta(0<\delta<\pi / 2)$

$$
\varlimsup_{r \rightarrow \infty} \frac{\log ^{+} \bar{n}^{l}\left(r, \Delta\left(\theta_{0}, \delta\right), \alpha\right)}{\log r}=\rho
$$

for any value of $\alpha$, with $l v-1$ possible exceptions.

Proof. By Theorem 2, there exists a sequence of filling disks of $w(z)$

$$
\Gamma_{n}:\left\{\left|z-z_{n}\right|<\left|z_{n}\right| \sigma_{n}\right\}, \quad\left|z_{n}\right|=r_{n}, \quad \sigma_{n} \rightarrow 0(n \rightarrow \infty)
$$

such that for any complex value $\alpha$,

$$
\bar{n}^{l)}\left(\Gamma_{n}, w=\alpha\right) \geq\left|z_{n}\right|^{\rho-\varepsilon_{n}},
$$

with some possible exceptions for $\alpha$ enclosed in $l v-1$ spherical circles with radius $r_{n}^{-\rho / 26}$ on the Riemann sphere, where $\varepsilon_{n} \rightarrow 0(n \rightarrow \infty)$.

Let $\theta_{0}$ be a cluster point of $\left\{\arg z_{n}\right\}$, then the direction $B: \arg z=\theta_{0}$ has the properties of Theorem 3. Otherwise, then there exists a positive number $\delta_{0}\left(0<\delta_{0}<\pi / 2\right)$ and $l v$ exceptional values $a_{j}(1 \leq j \leq l v)$ such that

$$
\varlimsup_{n \rightarrow \infty} \frac{\log \bar{n}^{l)}\left(r, \Delta\left(\theta_{0}, \delta_{0}\right), a_{j}\right)}{\log r}<\rho \quad(j=1,2, \ldots, l v) .
$$

On the other hand, since $\theta_{0}$ is a cluster point of $\left\{\arg z_{n}\right\}$, then there is a subsequence of $\left\{\arg z_{n}\right\}$ which converges to $\theta_{0}$. We may assume without loss of generality that $\lim _{n \rightarrow \infty} \arg z_{n}=\theta_{0}\left(0 \leq \theta_{0}<2 \pi\right)$. Hence for sufficiently large $n$ we have

$$
\Gamma_{n} \subset\left\{z ;\left|\arg z-\theta_{0}\right|<\delta_{0}\right\} .
$$

Let $\varepsilon_{0}=\min _{1 \leq l \neq J \leq l v}\left\{d\left(a_{i}, a_{j}\right)\right\}$, then $\varepsilon_{0}>0$. Note that $r_{n}^{-\rho / 26} \rightarrow 0$ $(n \rightarrow \infty)$, then

$$
r_{n}^{-\rho / 26}<\frac{\varepsilon_{0}}{2}
$$

when $n$ is large enough.

Because $\left\{\Gamma_{n}\right\}$ are a sequence of filling disks of $w(z)$, then there exists a subsequence $\left\{\Gamma_{n_{k}}\right\}$ of $\left\{\Gamma_{n}\right\}$ and an $a_{j_{0}} \in\left\{a_{j}\right\}_{j=1}^{v l}$ such that

$$
\bar{n}^{l)}\left(\Gamma_{n_{k}}, w=a_{j_{0}}\right) \geq r_{n_{k}}^{\rho-\varepsilon_{n_{k}}}
$$

Hence

$$
\begin{aligned}
& \varlimsup_{r \rightarrow \infty} \frac{\log \bar{n}^{l)}\left(r, \Delta\left(\theta_{0}, \delta_{0}\right), a_{j_{0}}\right)}{\log r} \\
& \geq \varlimsup_{k \rightarrow \infty} \frac{\log \bar{n}^{l)}\left(\Gamma_{n_{k}}, w=a_{j_{0}}\right)}{\log 2 r_{n_{k}}}
\end{aligned}
$$




$$
\begin{aligned}
& \geq \varlimsup_{k \rightarrow \infty} \frac{\log r_{n_{k}}^{\rho-\varepsilon_{n_{k}}}}{\log 2 r_{n_{k}}} \\
& =\rho .
\end{aligned}
$$

This is contrary to (22) and Theorem 3 is proved.

Acknowledgment. The author gratefully acknowledges the referees for their ardent corrections and valuable suggestions.

\section{REFERENCES}

[1] G. Valiron, Sur les directions de Borel des fonctions algébroïdes méromorphes d'ordre infinı, C. R. Acad. Sci. Parıs, 206 (1938), 735-737

[2] A. Rauch, Sur les algébroïdes entières, C. R. Acad. Scı. Parıs, 202 (1936), 2041-2043.

[ 3 ] N. Toda, Sur les directions de Julia et de Borel des fonctions algébroïdes, Nagoya Math. J., 34 (1969), 1-23.

[4] Y LÜ AND Y Gu, On the existence of Borel direction for algebroldal function, Kexue Tongbao (Science Bulletı), 28 (1983), 264-266.

[ 5 ] E. UllRich, Über den Einfluss der Verzweigtheit enner Algebroıde auf ihre Wertverteilung, J. Reine. Angew. Math., 167 (1932), 198-220.

[6] D. Sun, Man theorem on covering surfaces, Acta Math. Sc1. (Chinese), 14 (1994), 213-225.

[7] M. TsusI, Potential Theory in Modern Function Theory, Maruzen, Tokyo, 1959.

[8] Y Tumura, Quelques appiquations de la théorie de M. Ahlfors, Japan. J. Math., 18 (19421943), 303-322.

[9] D. Sun, Filling disks of algebrold functions, Chınese Ann. Math. Ser. A, 16 (1995), 238-243.

Department of APplied Mathematics

BeiJing University of Aeronautics and Astronautics

BEIJING, 100083

P. R. CHINA 